\title{
Evaluation of Biochemical Markers of Cardiac Risk in Antiretroviral Therapy (ART) -Treated HIV-Infected Adults in Rwanda
}

Marcus Bushaku ( $\sim$ marcusbushaku@gmail.com )

Regional Alliance for Sustainable Development (RASD)

\section{Caleb Nyamwange}

Moi University

\section{Arthur Kwena}

Moi University

Marc Twagirumukiza

Regional Alliance for Sustainable Development (RASD)

Jean Nepo Utumatwishima

Rwamagana Provincial Hospital

\section{Regine Mugeni}

Rwamagana Provincial Hospital

Victor Davila-Roman

Washington University in St. Louis

Aurore Nishimwe

University of Rwanda

Kenneth Schectman

Washington University in St. Louis, Division of Biostatistics

W.Todd. Cade

Duke University School of Medicine

\section{Dominic Reeds}

Division of Geriatrics and Nutritional Sciences

\section{Eugene Mutimura}

10. National Council for Science and Technology (NCST)

Research article

Keywords: CVD, HIV, Biomarkers, ART, Rwanda

Posted Date: March 22nd, 2022

DOI: https://doi.org/10.21203/rs.3.rs-1024775/v2 
License: (c) (i) This work is licensed under a Creative Commons Attribution 4.0 International License. Read Full License 


\section{Abstract}

Background: Similar to other African countries, life expectancy of people living with HIV infection has improved due to access to antiretroviral therapy (ART) in Rwanda. However, both HIV infection and use of ART are associated with cardiovascular disease (CVD) risks, due to adverse changes in some biochemical markers, causing dyslipidemia and other metabolic imbalances. Biomarkers for CVD risk in HIV-infected individuals taking ART, has not been well characterized in Rwanda. We evaluated the association between the use and duration of ART and biomarkers of CVD risk among HIV infected adults in Rwanda.

Methods: A total of 150 participants (18-45 years) from HIV clinics in public Health Center in Kigali included n=30 HIV-uninfected (HIV-) and n=120 HIV-infected (HIV+) adults. Among the HIV+ adults, $n=40$ participants were ART-naïve. Cross-sectional data were collected on health-related behaviors and biochemical markers of CVD risk. We compared CVD-related biochemical markers between HIV-, HIV+ ART-naïve and HIV+ on ART groups.

Results: The majority of participants were women ( $60 \%)$ and HIV- were younger ( $35 \pm 6$ vs. $31 \pm 6$ years). Total cholesterol and triglycerides concentrations were associated with ART usage. Serum triglycerides concentrations were lower in HIV+ ART-naïve compared to HIV+ on ART $(76.6 \pm 38.9 \mathrm{mg} / \mathrm{dl}$ vs. $85.0 \pm 38.3$ $\mathrm{mg} / \mathrm{dl} ; p<0.01)$. While total cholesterol concentrations was higher in HIV+ on ART than HIV+ ART-naïve $(136.0 \pm 45.1 \mathrm{mg} / \mathrm{dl}$ vs. $130.0 \pm 36.5 \mathrm{mg} / \mathrm{dl} ; \mathrm{p}<0.04), \mathrm{HDL}-\mathrm{C}$ was higher in those taking ART $(68.7 \pm 30.0$ $\mathrm{mg} / \mathrm{dl}$ vs. $55.0 \pm 25.7 \mathrm{mg} / \mathrm{dl} ; \mathrm{p}=0.02$ ) among HIV+ on ART for 0-6 months and 7-12 months respectively.

Conclusion: Higher serum total cholesterol and triglycerides were associated with use of ART in young adults with HIV. Although these values were within the upper limits of normal, our findings suggest early alterations in biomarkers of cardiac risk. These findings underscore the need for early evaluation of lipid profiles as biomarkers of cardiovascular disease risk, to effectively monitor how ART may contribute to cardiovascular disease and deter treatment programs in African countries.

\section{Background}

The number of people living with HIV infection continues to grow, and was estimated globally at 37.6 million [ 30.2 million- 45.0 million] in 2020 by UNAIDS [1]. In Rwanda, a recent population-based HIV impact assessment suggests a sustained general HIV prevalence of $3.0 \%$ among adults between 15 and 64 years over the last 4 years [2]. The prevalence is said to be higher in females (3.7\%) compared with males $(2.2 \%)$, and higher in urban (4.8\%) than rural areas (2.5\%) [3]. Similar to Western countries, the use of antiretroviral therapy (ART) has resulted in improved immunologic and virologic outcome and improved the wellbeing for HIV-infected individuals (HIV+), in Africa. However, the use of ART, particularly the first-generation antiretroviral medications has been associated with adverse metabolic disorders [4],[2] such as dyslipidaemia, hypertension and type 2 diabetes mellitus (T2D), which are risk factors for cardiovascular disease (CVD) [5],[6]. 
Whether the prevalence of CVD in people living with HIV on ART, is different from that in the general population, has been scarcely investigated in African countries [6]. Despite a lack of sufficient data on CVD in people living with HIV in Rwanda, studies at regional level have shown that the most affected are women living with HIV at a proportion of about $58 \%$ in Sub-Saharan Africa [7]. To address the question of if CVD risk is higher in individuals with HIV taking ART compared to HIV+ not taking ART and HIVuninfected individuals in Sub-Saharan Africa, specifically in Rwanda, we conducted a cross-sectional study to assess biomarkers of CVD risk in HIV+ on ART, HIV+ ART-naïve and HIV-negative in health centers in Rwanda.

\section{Methods}

\section{Study Design and Participants}

This was a health facility-based cross-sectional study conducted in seven health centers in an urban setting (Kigali city, Rwanda) serving as HIV infection clinics comprising Nyarugunga, Remera, Kinyinya, Masaka, Biryogo, Cornum, and Gikondo health Centers. Study participants included men and women aged between 18 and 45 years, and were either HIV-uninfected (-) or HIV-infected (+) on ART treatment or HIV+ ART-naïve. A total of 150 participants in the study included $30 \mathrm{HIV-uninfected} \mathrm{individuals} \mathrm{serving} \mathrm{as}$ the control group; 40 HIV-infected participants who were ART-naïve and 40 HIV-infected participants who were on WHO-recommended ART for 0-6 months, and 40 HIV-infected participants on ART for 7-12 months. Age and sex were considered during recruitment of study participants, to ensure good matching across each study group. Individuals presenting with active cardiac conditions, or significant symptoms of any heart disease such as hypertension (HTN), type 2 diabetes mellitus (T2D), or renal disease, and those with irregular ART adherence within 3 months prior to the recruitment period, were excluded from the study. Ethical approval was obtained from Institutional Research and Ethics Committee (IREC) at Moi Teaching and Referral Hospital (MTRH), Approval Number: 0003163, and from Rwanda National Ethics Committee (RNEC), FWA Assurance No.00001973; IRB 00001497 of IORG0001100. Written informed consent was obtained from all study participants.

\section{Study Procedure}

A structured questionnaire was used to collect data on social demographic characteristics (gender, age, marital status, residence, social economic status). Social economic status was defined based on Ubudehe categorization in Rwanda. In the Ubudehe approach, there are 5 categories (ABCD \& E) defined by a set of criteria, from the poorest category (without land, facing difficulties to have food) to the richer people [8]. Middle to high category $=$ monthly income $\geq 65,000$ Rwandan francs (RWF) (approximately 66 USD) to $\geq 600,000$ RWF ( 607 USD) and $\geq 780,000$ RWF (791 USD) to $\geq 7,200,000$ RWF (7,302 USD) annual income. Low Category $=\leq 45,000$ RWF (46 USD) to $\leq 65,000$ RWF (66 USD) per month and $\leq$ 540,000 RWF (548 USD) to $\leq 780,000$ RWF (791 USD) annual income [9]. Lifestyle (physical activity, smoking, alcohol consumption, eating habits) and clinical data (HIV clinical stage, high blood pressure (HBP). Elevated or high blood pressure (BP) was defined as the systolic BP of $140 \mathrm{mmHg}$ or higher, 
and/or diastolic BP of $90 \mathrm{~mm} \mathrm{Hg}$, and/or being on hypertensive medications [10]. Alcohol consumption as taking a drink that contained alcohol for the last 6 months [11]. Health diet defined based on how often are fruits and vegetables accompanying routine meals per week and unhealthy diet, defined as an impression of usually eating meals prepared with too much oil [12]. Where as physical activity participation was defined as vigorous-intensity exercise activities that cause large increases in heart rate like running, pedal cycle or football for at least 30 minutes continuously 3 times per week [13].

Blood specimens were collected for laboratory analysis of biochemical markers (NT-pro-BNP, hs-CRP, TC, HDL-C, LDL-C, TG, and glucose). Anthropometric measurements included weight and height, and body mass index (BMI) was calculated. BMI was calculated as weight in kilograms divided by height in meters $\left(\mathrm{Kg} / \mathrm{M}^{2}\right)$.

\section{Blood Sample Collection and Analysis}

Blood samples were collected in the morning under overnight fasting conditions of at least 8 hours. Coded blood specimens were transported to the WiWo global Indian specialized hospital laboratory in Kigali, for subsequent processing and analysis. NT-proBNP, hs-CRP, TC, HDL-C, LDL-C, TG, and glucose levels were measured. Serum dry tubes were centrifuged at 10, 000 relative centrifugal force (RCF) for 10 minutes. The centrifuged specimens with a surface lipid layer were transferred into secondary tubes for Cobas C-111 clinical lipid profile analysis (Roche Diagnostics). NT-pro BNP and hs-CRP were assayed using a Maglumi-600 automated machine. Surplus serum was stored at $-20^{\circ} \mathrm{C}$ and $-40^{\circ} \mathrm{c}$ to allow for repeat testing as needed.

\section{Data Management and Analysis}

The data obtained from both the questionnaire and biochemical assays, was cleaned, coded and stored in Microsoft Access. The data were imported into Statistical packages for social sciences (SPSS) for analysis (IBM Corp. Released 2020. IBM SPSS Statistics for Windows, Version 27.0. Armonk, NY: IBM Corp). Normal distribution was checked for all variables and for non-normally distributed data, nonparametric tests were used accordingly (variables: age, glucose, HDL, SBP, ProBNP, CRP and viral load). Both descriptive analyses, mean comparisons of biochemical markers analysis, and the relationship analysis between biochemical markers or patient characteristics and ART were performed. Significance difference was always considered for a p-value $<0.05$ with corresponding $95 \%$ confidence interval.

\section{Results}

\section{General Characteristics of Study Participants}

The majority of the participants were female (60\%). The HIV-uninfected (HIV-) participants were younger than HIV-infected (HIV+) participants ( $35 \pm 6$ vs. $31 \pm 6$ ), and $44 \%$ of HIV-participants were young, aged between 26 and 33 years with mean age of $32( \pm 6)$ years. Most participants were city dwellers (83.3\% vs. $16.7 \%)$. There was no difference between the proportion of participants between those with low or middle to high social economic status (Table 1). Table 1 also summarizes the clinical and lifestyle 
characteristics of study participants. Majority of participants were in HIV stage I of the WHO HIV classification compared to those in HIV Stage 2 and above ( $82.5 \%$ vs. $17.5 \%$ ) and none were either in HIV stage 3 or Stage 4 of illnesses. In addition, there was no difference in the proportion of participants with HIV Stage 1 or HIV Stage 2 for HIV+ ART-naïve and HIV+ on ART for 6 months or 12 months. Although the majority of participants had a normal BMI of $18.5-24.9$ in $\mathrm{Kg} / \mathrm{M}^{2}$ of $70 \%$ compared with the rest of participants, the HIV+ participants group had a significantly higher proportion of those with $\mathrm{BMI}$ in $\mathrm{Kg} / \mathrm{M}^{2}$ less than 18.5 (Table 1). Although there was no difference between those with normal and high Blood Pressure (BP) between HIV- and HIV+ ART-naïve or on ART, 7.3\% of participants were diagnosed with high $\mathrm{BP}$, and the majority had normal Blood Pressure (BP) (92.7\% vs. 7.3\%). The HIV- participants were less likely to smoke compared to HIV+ (100\% vs. $73.3 \%)$, and were less likely to consume alcohol $(50 \%$ vs. $30.8 \%$ ) with the overall majority of the participants reported to have had very limited physical activity $(63.3 \%$ vs. $36.7 \%)$ (Table 1$)$. 
Table 1

Participants' demographics, clinical and lifestyle characteristics

\begin{tabular}{|c|c|c|c|c|c|}
\hline \multirow[t]{4}{*}{ Variable } & HIV- $(n=30)$ & HIV+ & HIV+ & HIV+ & Total \\
\hline & \multirow[t]{3}{*}{$\mathrm{n}(\%)$} & $\begin{array}{l}\text { ART-naïve } \\
(n=40)\end{array}$ & On ART & on ART & \multirow[t]{3}{*}{$n(\%)$} \\
\hline & & $n(\%)$ & $\begin{array}{l}0-6 \text { months } \\
(n=40)\end{array}$ & $\begin{array}{l}7-12 \text { months } \\
(n=40)\end{array}$ & \\
\hline & & & n (\%) & n (\%) & \\
\hline \multicolumn{6}{|l|}{ Gender } \\
\hline Male & $14(46.70)$ & $17(42.50)$ & 15 (37.50) & $14(35.00)$ & $60(40.00)$ \\
\hline Female & 16 (53.30) & $23(57.50)$ & $25(62.50)$ & $26(65.00)$ & $90(60.00)$ \\
\hline $\begin{array}{l}\text { Age (years) } \\
\text { (Mean } \pm \text { SD) }\end{array}$ & $35 \pm 6$ & $30 \pm 6$ & $31 \pm 6$ & $33 \pm 7$ & $32 \pm 6$ \\
\hline \multicolumn{6}{|l|}{ Residence } \\
\hline Rural & $2(6.70)$ & $11(27.50)$ & $4(10.00)$ & $8(20.00)$ & 25 (16.70) \\
\hline Urban & 28 (93.30) & $29(72.50)$ & $36(90.00)$ & $32(80.00)$ & $\begin{array}{l}125 \\
(83.30)\end{array}$ \\
\hline \multicolumn{6}{|c|}{$\begin{array}{l}\text { Social Economic } \\
\text { Status }^{1}\end{array}$} \\
\hline Low & 11 (36.70) & $26(65.00)$ & $24(60.00)$ & $22(55.00)$ & $83(55.40)$ \\
\hline Middle to high & 19 (68.30) & $14(35.00)$ & $16(40.00)$ & $18(45.00)$ & 67 (44.70) \\
\hline \multicolumn{6}{|l|}{ WHO stage } \\
\hline Stage 1 & & $36(90.00)$ & $32(80.00)$ & 31 (77.50) & 99 (82.50) \\
\hline Stage $2+$ & & $4(10.00)$ & $8(20.00)$ & $9(22.50)$ & $21(17.50)$ \\
\hline $\begin{array}{l}\mathrm{BMI} / \mathrm{kg} / \mathrm{m}^{2} \\
(\text { Mean } \pm \mathrm{SD})\end{array}$ & $23.94 \pm 3.44$ & $22.98 \pm 3.68$ & $22.89 \pm 3.61$ & $22.33 \pm 3.39$ & $22.97 \pm 3.55$ \\
\hline \multicolumn{6}{|l|}{$\begin{array}{l}\text { Blood pressure } \\
\text { (BP) }\end{array}$} \\
\hline Normal & $29(96.70)$ & $39(97.50$ & $34(85.00)$ & $37(92.50)$ & $\begin{array}{l}139 \\
(92.70)\end{array}$ \\
\hline $\mathrm{High}^{2}$ & $1(3.30)$ & $1(2.50)$ & $6(15.00)$ & $3(7.50)$ & $11(7.30)$ \\
\hline \multicolumn{6}{|l|}{ Smoking status } \\
\hline Yes & $0(0.00)$ & $14(35.00)$ & $8(20.00)$ & $10(25.00)$ & $32(21.30)$ \\
\hline
\end{tabular}




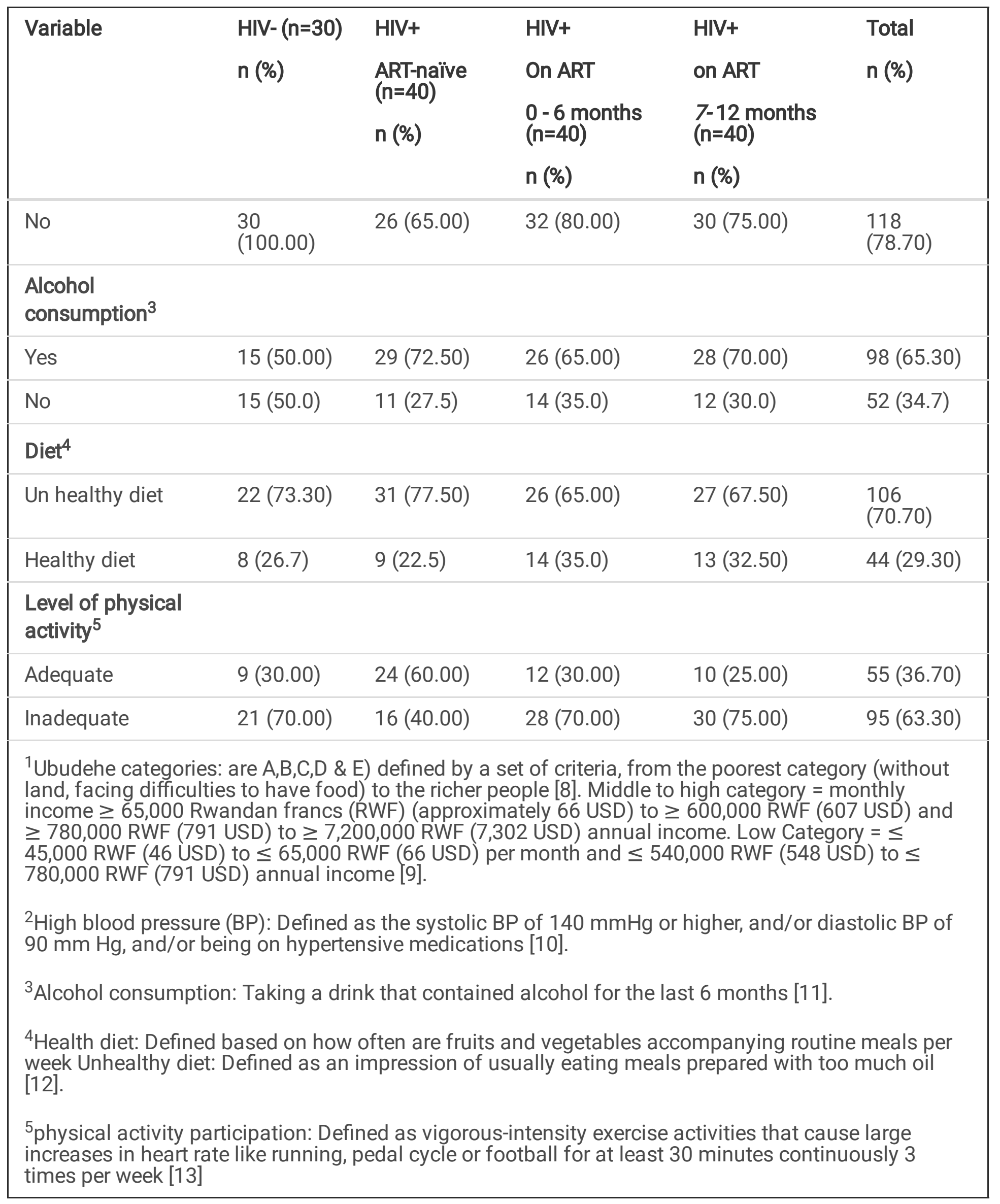

\section{Biochemical characteristics of study participants}


Analysis of serum biochemical markers of study participants suggest that generally the serum biochemical markers were higher among HIV-participants compared to HIV+ participants (Table 2a) and more higher levels being among those on ART versus ART naïve. The overall changes were observed in all biomarkers however; almost all the serum levels were within the cutoff values (normal range of each marker). 
Table 2

a: Comparison between HIV+ and HIV-participants

\begin{tabular}{|c|c|c|c|}
\hline Variables & $H I V+(n=120)$ mean $\pm S D$ & $\begin{array}{l}H I V-(n=30) \\
\text { mean } \pm S D\end{array}$ & $\begin{array}{l}p- \\
\text { value }\end{array}$ \\
\hline Age & $31 \pm 7$ & $35 \pm 6$ & $0.01 *$ \\
\hline $\mathrm{BMI} \mathrm{kg/m2}$ & $22.70 \pm 3.50$ & $23.90 \pm 3.40$ & 0.09 \\
\hline $\mathrm{HDL}-\mathrm{C}(\mathrm{mg} / \mathrm{dl})$ & $59.10 \pm 27.00$ & $62.80 \pm 20.40$ & $0.47 *$ \\
\hline LDL-C (mg/dl) & $57.00 \pm 22.50$ & $61.40 \pm 23.00$ & 0.34 \\
\hline Total Cholesterol (mg/dl) & $130.30 \pm 43.00$ & $144.60 \pm 31.40$ & 0.09 \\
\hline CRP (ng/dl) & $12976.20 \pm 27508.20$ & $2038.20 \pm 2881.70$ & $0.04 *$ \\
\hline Fasting Blood glucose (mg/dL) & $107.00 \pm 21.00$ & $107.00 \pm 21.00$ & 0.87 \\
\hline Triglycerides(mg/dl) & $77.10 \pm 34.80$ & $97.20 \pm 48.90$ & 0.01 \\
\hline $\begin{array}{l}\text { Serum plasma level of NT- } \\
\text { ProBNP in } \mathrm{pg} / \mathrm{ml}\end{array}$ & $96.90 \pm 112.80$ & $137.60 \pm 82.10$ & $<0.01^{*}$ \\
\hline \multicolumn{4}{|c|}{ Table 2b: HIV-infected ART-naïve versus HIV-infected on ART } \\
\hline & $\begin{array}{l}\text { HIV+ ART-naïve }(n=40) \\
\text { mean } \pm S D\end{array}$ & $\begin{array}{l}\text { HIV+ on ART }(n=80) \\
\text { mean } \pm S D\end{array}$ & $\begin{array}{l}\mathrm{p}- \\
\text { value }\end{array}$ \\
\hline Age & $32 \pm 7$ & $32 \pm 6$ & $0.89 *$ \\
\hline BMI & $23.40 \pm 3.60$ & $22.60 \pm 3.50$ & 0.59 \\
\hline $\mathrm{HDL}-\mathrm{C}(\mathrm{mg} / \mathrm{dl})$ & $57.50 \pm 22.10$ & $61.80 \pm 28.60$ & $0.70 *$ \\
\hline LDL-C (mg/dl) & $57.30 \pm 20.70$ & $58.30 \pm 24.20$ & 0.36 \\
\hline Total Cholesterol (mg/dl) & $130.00 \pm 36.50$ & $136.00 \pm 45.10$ & 0.04 \\
\hline CRP (ng/dl) & $11194.00 \pm 26251.90$ & $10433.80 \pm 24021.50$ & $0.75^{\star}$ \\
\hline Fasting Blood glucose (mg/dL) & $106.00 \pm 22.00$ & $108.00 \pm 21.00$ & 0.58 \\
\hline Triglycerides $(\mathrm{mg} / \mathrm{dl})$ & $76.60 \pm 38.90$ & $85.00 \pm 38.30$ & $<0.01$ \\
\hline $\begin{array}{l}\text { Serum plasma level of NT- } \\
\text { ProBNP in } \mathrm{pg} / \mathrm{ml}\end{array}$ & $111.60 \pm 95.40$ & $99.20 \pm 118.90$ & $0.25^{\star}$ \\
\hline \multicolumn{4}{|c|}{ Table 2c: Comparison between HIV+ on ART for 0-6 months vs. HIV+ on ART for 7-12 months } \\
\hline & $\begin{array}{l}\text { HIV+ on ART } \\
(0-6 \text { months }) \\
(n=40) \text { mean } \pm S D\end{array}$ & $\begin{array}{l}\text { HIV+ on ART } \\
(7-12 \text { months })(n=40) \\
\text { mean } \pm S D\end{array}$ & $\begin{array}{l}\mathrm{p}- \\
\text { value }\end{array}$ \\
\hline
\end{tabular}




\begin{tabular}{|c|c|c|c|}
\hline Variables & $H I V+(n=120)$ mean $\pm S D$ & $\begin{array}{l}\text { HIV- }(n=30) \\
\text { mean } \pm S D\end{array}$ & $\begin{array}{l}p- \\
\text { value }\end{array}$ \\
\hline Age & $31 \pm 6$ & $33 \pm 7$ & $0.29 *$ \\
\hline BMI & $22.90 \pm 3.60$ & $22.33 \pm 3.40$ & 0.47 \\
\hline $\mathrm{HDL}-\mathrm{C}(\mathrm{mg} / \mathrm{dl})$ & $68.70 \pm 30.00$ & $55.00 \pm 25.70$ & $0.02^{\star}$ \\
\hline LDL-C (mg/dl) & $59.40 \pm 27.60$ & $57.20 \pm 20.70$ & 0.68 \\
\hline Total Cholesterol (mg/dl) & $143.90 \pm 45.90$ & $128.10 \pm 43.30$ & 0.12 \\
\hline Triglycerides(mg/dl) & $81.50 \pm 31.90$ & $88.50 \pm 43.90$ & 0.42 \\
\hline $\mathrm{CRP}(\mathrm{ng} / \mathrm{dl})$ & $10160.30 \pm 20472.30$ & $10707.20 \pm 27378.80$ & $0.77^{*}$ \\
\hline Fasting Blood glucose (mg/dL) & $107.00 \pm 22.00$ & $109.00 \pm 20.00$ & 0.59 \\
\hline $\begin{array}{l}\text { Serum plasma level of NT- } \\
\text { ProBNP in } \mathrm{pg} / \mathrm{ml}\end{array}$ & $105.40 \pm 148.30$ & $93.00 \pm 81.20$ & $0.69 *$ \\
\hline
\end{tabular}

While evaluating the correlation between HIV status, use and duration of ART among HIV+ participants, and changes in biochemical markers, a positive correlation was observed between all biomarkers and the use of ART (Table 2). Differences were observed for serum triglycerides, and in serum total cholesterol with a strong significant positive correlation between triglycerides among HIV+ who were ART-naïve vs. $\mathrm{HIV+}$ on ART [76.6 $( \pm 38.9) \mathrm{mg} / \mathrm{dl}$ vs. $85.0( \pm 38.30) \mathrm{mg} / \mathrm{dll}$; $\mathrm{p}<0.01$ and serum total cholesterol were significantly higher in HIV+ on ART than HIV+ who were ART-naïve $[130.0( \pm 36.50) \mathrm{mg} / \mathrm{dl}$ vs. $136.0( \pm 45.10) \mathrm{mg} / \mathrm{dll} ; \mathrm{p}<0.04$ (Table $2 \mathrm{~b}$ ). Comparisons between HIV+ on ART for $0-6$ months vs. HIV+ on ART for 7-12 months showed that lower HDL-C was significantly associated with longevity of exposure to ART therapy $68.7( \pm 30.0) \mathrm{mg} / \mathrm{dl}$ vs. $55.0( \pm 25.70) \mathrm{mg} / \mathrm{dll}$; $p=0.02$ (Table $2 \mathrm{c})$.

\section{Diet and physical lifestyle covariates in the study participants}

Analysis results of diet and physical activity in study participants are not very conclusive. The physical activity classified by WHO category in low or adequate physical activity. The analysis of inflammatory biomarkers versus physical activity revealed that the inflammation is always lower in a group of participants with adequate physical activity but not statistically significant. The diet habits classified by health diet, defined based on how often are fruits and vegetables accompany routine meals per week and unhealthy diet defined as an impression of usually eating meals prepared with too much oil. The analysis of CVD risk biomarkers versus diet revealed that the HDL-C levels were lower in a group of health diet related habit but not statistically significant.

\section{Discussion}


Our analysis evaluated the association of abnormal changes in biochemical markers of cardiovascular risk among HIV-infected adults on antiretroviral therapy compared to HIV-infected adults who were antiretroviral therapy-naïve. Furthermore, we assessed whether duration of antiretroviral therapy use was associated with adverse abnormal changes in biochemical markers of cardiovascular risk for a duration of 0-6 months compared to $7-12$ months. This study did not investigate individual ART molecule, individual drug or individual therapy regimen. Our study showed that ART treated patients had significantly high levels of Total Cholesterol and Triglycerides as compared to ART-naïve patients. The proportion of patients with dyslipidaemia among our ART treated participants was higher than the rate reported in a study from Cameroon and rural Uganda [14][15]. There are suggestions that the magnitude of first-line ART-induced lipid imbalances could vary across populations and settings. Based on LDL-C cut-off values, dyslipidemia in our study was common and higher than that reported in a study from Cameroon [14], Western India (30\%) [16] and Uganda (6\%) [15]. However, the prevalence of high LDL-C prior to ART (5\%) was almost same as reported in Uganda and Western India (4\%) [17], but less than that reported in Cameroon (21\%) [14]. Similar to the Cameroon study [14], our study showed no changes in HDL-C levels after ART, which is not in accordance with the findings in Western India [17], which showed significant increase in HDL-C after 18 months of treatment with first-line ART regimen.

The association between HIV infection and use of antiretroviral therapy with cardiometabolic disease and biochemical biomarkers of cardiovascular risk has been documented in several settings in developed countries [18] and in Rwanda [19]. However, to the best of our knowledge, this is the first study in Rwanda to explore the relationship between ART and biochemical biomarkers of CVD risk in patients with HIV infection in comparison with HIV-uninfected adults.

Current data suggest that both HIV infection and antiretroviral therapy are associated with, and may increase the risk of cardiovascular disease, a consequence of changes in specific biochemical biomarkers of CVD risk, mainly associated with dyslipidemia [20],[21]. Our findings are consistent with a systematic review and meta-analysis that reported that among antiretroviral therapy treated HIV-infected adults in sub-Saharan Africa, use of ART was associated with higher LDL- Cholesterol and low HDL-Cholesterol [22]. Thus, similar biochemical markers of cardiac risk were reported, and the study suggested that the differences in cardiometabolic traits between HIV-infected and uninfected individuals were associated with HIV infected and use of ART [22]. Similarly, in agreement with our findings, one study in Tanzania reported that HIV-infected adults on antiretroviral therapy who presented a cluster of biomarkers of cardiovascular risk had a higher lifetime cardiovascular risk than HIV-uninfected adults [23].

The present study was undertaken to identify whether potential candidate serum biochemical biomarkers of CVD were influenced by ART in HIV-infected adults in Rwanda. In addition, CVD risk among study participants was examined to ascertain whether ART predisposes to an overall increased risk of CVD. Notably, more than a half of the study participants were female. Although females may be presenting more frequently in HIV clinics and seeking healthcare services more than males, women between 24-55 years in sub-Saharan Africa are twice more likely to be living with HIV infection than men in the same age 
group. Our findings concur with those reported by global systematic review [20], and by other subSaharan Africa data [21].

The majority of participants were in young age range between 26 and 33 years, and the mean age was similar among all study groups. Younger age constitutes a lower risk of CVD in various studies conducted in Africa, Europe and other countries where increased age is a predictor of CVD mortality, and higher prevalence of metabolic associated diseases [24],[25]. Most study participants resided in an urban setting. Individuals living in most African urban settings are more likely to consume unhealthy foods containing high animal fats with low fiber and vitamins resulting in increased the risk of CVD. Among study participants $16 \%$ and $5.3 \%$ were overweight and obese, respectively. These figures are higher than those reported in the previous STEP Survey in Rwanda, which reported $14.3 \%$ and $2.8 \%$ as overweight and obese, respectively [26]. Weight gain is common in HIV-infected adults who initiate ART treatment, which if not controlled may result in adipose tissue changes. Studies from developed countries indicate that excess adiposity and HIV infection contribute to metabolic complications [27]. Additionally, this study shows potential difference in patients profiles (BMI, Lifestyles) in relation to cardiovascular risk between the high income countries (HICs) and low-and-middle income countries (LMICs), where in this study patients starting ART are lean and have relatively lower BMI (23.94 \pm 3.44$)$. This finding contrast with reported data from high income countries (HICs) [28] The majority of participants over $60 \%$ lived a sedentary life style. Although this figure is higher than that reported in the STEP Survey among the general population, it is similar to that reported in Kigali in the STEP Survey, which showed that only $46.7 \%$ were highly active [27]. Most of study participants had an unhealthy diet with fat saturated rich animal foods with low fiber and vitamins. These results concur with the findings of the STEP Survey, which showed that most participants did not consume fruit and vegetables [25].

Comparison of biochemical markers between HIV-infected and HIV-Uninfected adults participants suggest abnormal serum levels of HDL- cholesterol, LDL- cholesterol, total cholesterol and triglycerides. Low HDL levels were observed in ART-treated participants between 7 to 12 months compared to ARTtreated participants between 0 and 6 months. HDL-C is considered a cholesterol scavenger that removes excess cholesterol from the blood. Low HDL-C constitutes a risk of hypercholesterolemia and this constitutes an increased risk of CVD. Our findings are similar to one study in Cameroon which assessed the prevalence and characteristics of lipid profile derangements associated with antiretroviral therapy [14]. In this study, it was observed that use of ART was significantly and positively associated with high total cholesterol, LDL-cholesterol and HDL-cholesterol [14]

The changes in HDL-C were also observed in a Tanzanian study [29], as well as other studies in the US and Europe [30],[31], indicating the need for routine clinical monitoring and care of HIV patients to ensure timely prevention and management of any cardiovascular disease associated derangements and risks. Hypercholesterolemia and hypertriglyceridemia were characteristic of dyslipidemia in the present study with higher serum plasma levels in ART treated patients and statistically significant. Similar findings were reported from an earlier study [32],[30]. There was no statistically significant differences observed for serum plasma levels of HDL-C, LDL-C, hs-CRP, NT-ProBNP and glucose, between ART naïve and ART 
treated participants. There was a significant positive correlation between triglycerides $(p<0.01)$, cholesterol $(p<0.04)$ and use of antiretroviral therapy. Similar results were reported elsewhere [33]. In line with our findings, though many studies have failed to demonstrate that ART is the main cause of most derangements in lipid metabolism, a study by Cunha et.al,(2015) demonstrated that PI-based ART combination impairs normal lipid metabolism resulting in increased triglyceride levels and lowered HDL-C [34].

Several developed countries and low- and middle-income countries (LMICs) such as Brazil as well as Rwanda have registered tremendous HIV treatment progress in terms of immunologic and virologic outcome after access to potent ART [35][ [36]. The efficacy and safety of ART has substantially improved the wellbeing of people living with HIV infection after the introduction of newer drug classes of antiretrovirals that are now available to patients and HIV care providers. Thus, constant evaluation and assessment of potential metabolic derangements is required in order to curtail potential adverse impact of HIV treatment and functional cure towards the economic sustainability of patients living with HIV infection.

Our study has some limitations. First, it is a cross sectional study which limits temporal trends and patterns in association that would provide more evidence to causation. Second, our participants in the study were conveniently enrolled from Health Care Clinics in urban areas of the City of Kigali province limiting inclusion of diversity of participants from rural areas of Rwanda. Third, our sample size is relatively small, which makes it rather explorative and calling for a lager study to confirm our conclusions. Finally, although data were presented for HIV-infected adults on antiretroviral therapy for 0-6 months and for 7-12 months, ideally limited changes may happen before the 4th month of antiretroviral therapy.

\section{Conclusions}

In conclusion, our study indicated that changes in serum levels of total cholesterol and triglycerides were associated with use of ART in HIV-infected adults on treatment in Rwanda. Compared to data from developed countries, these biochemical markers of cardiac risk changes were within the upper limits of normal ranges. Therefore, these findings likely revealed early increases in both biomarkers accompanying antiretroviral therapy. In countries faced with the scourge of HIV infection, routine clinical monitoring of lipid profile and other cardiovascular risk factors should be under the treatment guidelines to be monitored in patients on antiretroviral therapy so that any adverse effects of HIV treatments can be optimally managed. Our study suggests the need for early evaluation of lipid profiles as biomarkers of cardiac risk, to effectively monitor how antiretroviral therapy may contribute to cardiovascular disease risk, and deter treatment programs in Rwanda and other African countries.

\section{Abbreviations}

ART

Antiretroviral therapy 
CAD

Coronary artery disease

CVD

Cardiovascular Diseases

HAART

Highly Active Antiretroviral Therapy

HDL-C

High density lipoproteins

cholesterol

IREC

Institutional Research and Ethics Committee

LDL-C

Low Density Lipoprotein cholesterol

RNEC

Rwanda National Ethics Committee

TC

Total cholesterol

TG

Triglycerides

\section{Declarations}

Ethics approval and consent to participants: This study was approved by Institutional Research and Ethics Committee (IREC) at Moi Teaching and Referral Hospital (MTRH) (approval notice: 0003163) and by Rwanda National Ethics Committee (RNEC), FWA (Assurance No.00001973; IRB 00001497 of IORG0001100). Participants were informed of the potential risks and benefits of the study and signed an informed consent form in Kinyarwanda language before enrolment in the study. All participant data were anonymized to optimize privacy and confidentiality of responses.

Consent for publication: Not Applicable

Data statement: Data is available upon request to the corresponding author

Competing Interests statement: The authors declare that they have no competing interests

Funding: This study was fully funded by Regional Alliance for Sustainable Development (RASD), Rwanda a non-government organization through its D43 NIH funding \#D43TW010335, for the scholarship offered to pursue postgraduate Master Degree studies in Medical Biochemistry.

Author's Contributions: MB, CN, AK, EM: Protocol concept, study design, laboratory analysis, data collection, data analysis interpretation and drafted initial version of manuscript. MT, JNU, RM, VD, DR, KS, 
AN, TC, EM: Adjudication of the data analysis and review of the whole manuscript. All authors read, contributed to and approved the final manuscript.

Acknowledgements: We are grateful to Dr. Rajesh vedanthan, Dr. Chris Longenecker, Dr. Gerald S. Bloomfield, Mrs. Lamberte Umurerwa, Dr. John Nyirigira, Dr. Karengera Steven and Dr. Jean Damascene Kabakambira who provided guidance while formulating the concept of this work. We gratefully acknowledge Professor Eleanor N Fish, PhD, for her writing assistance while in manuscript preparation. We thank Mr. Prashanta Dash, center head and laboratory supervisor at WiWo Global Indian Specialized Hospital, Dushimimana Jacques and Eric lyamuremye both lab technicians, for their contributions in laboratory analysis. We extend thanks to all participants of the study.

Authors' information: ${ }^{1}$ Regional Alliance for Sustainable Development (RASD), Kigali, Rwanda, Biochemistry and Clinical Chemistry, School of Medicine, Moi University, Eldoret, Kenya. ${ }^{2}$ Biochemistry and Clinical Chemistry, School of Medicine, Moi University, Eldoret, Kenya. ${ }^{3}$ Biochemistry and Clinical Chemistry, School of Medicine, Moi University, Eldoret, Kenya. ${ }^{4}$ Regional Alliance for Sustainable Development (RASD), Kigali, Rwanda, Faculty of Medicine and health Sciences, Ghent University, Ghent, Belgium. ${ }^{5}$ Rwamagana Provincial Hospital, Rwamagana, Eastern province, Rwanda. ${ }^{6}$ Rwamagana Provincial Hospital, Rwamagana, Eastern province, Rwanda. ${ }^{7}$ Cardiovascular division, Departement of Medicine, Washington University in St. Louis, MO, US. ${ }^{8}$ Center for Human Nutrition, Division of Geriatrics and Nutritional Sciences, Department of Medicine, St. Louis, MO, US. ${ }^{9}$ Washington University in St. louis, Division of Biostatistics, St. Louis, MO, US. ${ }^{10}$ University of Rwanda, College of Medicine and Health Sciences (UR-CMHS), Kigali, Rwanda. ${ }^{11}$ Duke University School of Medicine, 27710, Durham, NC, US.

${ }^{12}$ National Council for Science and Technology (NCST), Kigali, Rwanda.

\section{References}

1. Statistics GH, “FACT SHEET,” no. June, 2021.

2. H. I. A. RP-B. R. 2018-2019, "RWANDA POPULATION-BASED HIV IMPACT ASSESSMENT," no. October 2019, pp. 2-7.

3. Sabin N. "Rwanda Population-based HIV Impact Assessment (RPHIA) - Key findings," vol. 1, no. December, 2019.

4. Bloomfield GS, Velazquez EJ. "Hiv and cardiovascular disease in sub-saharan africa: The sutton law as applied to global health," Journal of the American College of Cardiology, vol. 61, no. 23. p. 2395, 11-Jun-2013.

5. Alinda K, Grobusch. "HIV and risk of cardiovascular disease in sub-Saharan Africa. Rationale and design of the Ndlovu Cohort Study; 2017.

6. Mutimura S, "The Human Immunodeficiency Virus and the CardiometabolicSyndrome in the Developing World: An African Perspective," vol. 3, no. 2, pp. 106-110, 2015. 
7. Vos A, et al. HIV and risk of cardiovascular disease in sub-Saharan Africa: Rationale and design of the Ndlovu Cohort Study Background and rationale. Eur J Prev Cardiol. 2017;24(10):1043-50.

8. I. developed in Rwanda. "Innovation developed in Rwanda to support social protection interventions," pp. 2-3, 2004.

9. “PERCEPTION OF HOUSEHOLD, MEMBERS ON. UBUDEHE CATEGORIES IN,” 2013.

10. Guidelines CP. CLINICAL PRACTICE GUIDELINES Management of Hypertension, vol. 13.

11. Ikeda MLR, et al., "Association of Blood Pressure and Hypertension with Alcohol Consumption in HIVInfected White and Nonwhite Patients," vol. 2013, 2013.

12. Lachat $C$, et al., "Diet and Physical Activity for the Prevention of Noncommunicable Diseases in Lowand Middle-Income Countries: A Systematic Policy Review," vol. 10, no. 6, 2013.

13. Trapp EG, Chisholm DJ, Freund J, Boutcher SH. "The effects of high-intensity intermittent exercise training on fat loss and fasting insulin levels of young women," pp. 684-691, 2008.

14. Pefura Yone EW, Betyoumin AF, Kengne AP, Kaze Folefack FJ, Ngogang J. First-line antiretroviral therapy and dyslipidemia in people living with HIV-1 in Cameroon: A cross-sectional study. AIDS Res Ther. 2011;8:1-8.

15. Buchacz K, et al. Changes in lipid profile over 24 months among adults on first-line highly active antiretroviral therapy in the Home-Based AIDS Care Program in Rural Uganda. J Acquir Immune Defic Syndr. 2008;47(3):304-11.

16. Kalyanasundaram AP, Jacob SM, Hemalatha R, Sivakumar MR. Prevalence of lipodystrophy and dyslipidemia among patients with HIV infection on generic ART in rural South India. $J$ Int Assoc Physicians AIDS Care. 2012;11(5):329-34.

17. Indumati V, Vijay V, Shekhanawar MS, Rajeshwari M, Amareshwaras, Shantala D. Comparison of serum lipid profile in hiv positive patients on art with art naïve patients. J Clin Diagnostic Res. 2014;8(10):CC06-9.

18. Mutimura E, Stewart A, Rheeder P, Crowther NJ. Metabolic function and the prevalence of lipodystrophy in a population of HIV-infected African subjects receiving highly active antiretroviral therapy. J Acquir Immune Defic Syndr. 2007;46(4):451-5.

19. Mutimura E, et al. Insulin resistance change and antiretroviral therapy exposure in HIV-infected and uninfected Rwandan women: A longitudinal analysis. PLoS One. 2015;10(4):1-12.

20. Silva B, Peixoto G, Luz S, Moraes S, Peres S, "Adverse effects of chronic treatment with the Main subclasses of highly active antiretroviral therapy: a systematic review," HIV Med., vol. 20, no. 7, pp. 429-438, Aug. 2019.

21. Dillon DG, "Association of HIV and ART with cardiometabolic traits in sub-saharan africa: A systematic review and meta-analysis [Int J Epidemiol, 42 (2013)(1754-1771)] DOI: 10.1093/ije/dyt198," International Journal of Epidemiology, vol. 45, no. 6. Oxford University Press, pp. 2210-2211, Dec-2016. 
22. Dillon DG, "Association of HIV and ART with cardiometabolic traits in sub-saharan africa: A systematic review and meta-analysis [Int J Epidemiol, 42 (2013)(1754-1771)] DOI: 10.1093/ije/dyt198," Int. J. Epidemiol., vol. 45, no. 6, pp. 2210-2211, 2016.

23. Kingery JR, et al. Short-term and long-term cardiovascular risk, metabolic syndrome and HIV in Tanzania. Heart. 2016;102(15):1200-5.

24. Tsai MC, Lee CC, Liu SC, Tseng PJ, Chien KL, "Combined healthy lifestyle factors are more beneficial in reducing cardiovascular disease in younger adults: a meta-analysis of prospective cohort studies," Sci. Rep., vol. 10, no. 1, p. 18165, Dec. 2020.

25. Risk D, Report F. "REPUBLIC OF RWANDA Rwanda Non-communicable," no. November, 2015.

26. “REPUBLIC OF RWANDA. Rwanda Non-communicable Diseases Risk Factors Report,” 2015.

27. Ministry of Health (Rwanda). "WHO Stepwise approach to NCD surveillance. Rwanda Noncommunicable Diseases Risk Factors Report," no. November, 2015.

28. Mounzer K, et al. Changes in Body Mass Index Associated with Antiretroviral Regimen Switch Among Treatment-Experienced, Virologically Suppressed People Living with HIV in the United States. AIDS Res Hum Retroviruses. 2021;37(11):852-61.

29. Ombeni W, Kamuhabwa AR. "Lipid Profile in HIV-Infected Patients Using First-Line Antiretroviral Drugs," vol. 15, no. 2, pp. 164-171, 2016.

30. Nsagha DS, et al., "Risk Factors of Cardiovascular Diseases in HIV/AIDS Patients on HAART," Open AIDS J., vol. 9, no. 1, pp. 51-59, Oct. 2015.

31. Id LA, Namwongprom S, Sudjaritruk T. "Metabolic syndrome, biochemical markers, and body composition in youth living with perinatal HIV infection on antiretroviral treatment," vol. 43, pp. 1-13, 2020.

32. Zhou DT, et al., "Changes in lipid profiles of HIV+ adults over nine months at a Harare HIV clinic: A longitudinal study," Biochem. Res. Int., vol. 2016, no. April 2004, 2016.

33. N. A. et. a. Nsagha, "Risk Factors of Cardiovascular Diseases in HIV/AIDS Patients on HAART.," Open AIDS J., vol. 9, no. June 2012, pp. 51-59, 2015.

34. Cunha J, et al., "new drugs," vol. 4, no. 2, pp. 56-77, 2015.

35. Benzaken AS, Pereira GFM, Costa L, Tanuri A, Santos AF, Soares MA. Antiretroviral treatment, government policy and economy of HIV/AIDS in Brazil: Is it time for HIV cure in the country? AIDS Res Ther. 2019;16(1):1-7.

36. Ford $\mathrm{N}$, et al. Benefits and risks of rapid initiation of antiretroviral therapy. Aids. 2018;32(1):17-23. 